\title{
Isokinetic performance of ankle evertor and invertor muscles in adolescent female volleyball athletes
}

\author{
Leandro Viçosa Bonetti ${ }^{1}$, Nicole Coulon Grisa ${ }^{1}$, Juliete Palandi ${ }^{2}$, André Luis Temp Finger ${ }^{3}$, Thiago De Marchi ${ }^{3}$, \\ Gerson Saciloto Tadiello ${ }^{1}$ \\ ${ }^{1}$ Universidade de Caxias do Sul, UCS, Department of Physical Therapy, Caxias do Sul, RS, Brazil; ${ }^{2}$ Universidade \\ Federal de Santa Catarina, UFSC, Departamento de Bioquímica, Florianópolis, SC, Brazil; ${ }^{3}$ Faculdade \\ Cenecista Bento Gonçalves, CNEC, Department of Physical Therapy, Bento Gonçalves, RS, Brazil.
}

\begin{abstract}
Aims: This study aimed to analyze the muscular performance of evertor and invertor ankle muscles of adolescent volleyball athletes. Methods: The information provided by database concerning the isokinetic evaluation of ankle muscles from 20 female volleyball players between the ages of 14-17 years old were analyzed. The isokinetic dynamometer was used in a concentric-concentric mode for the ankle evertor and invertor muscles at angular velocities of $60^{\circ} / \mathrm{s}$, and $180^{\circ} \mathrm{s}$. Results: Seven athletes had suffered ankle sprains (6 affected the non-dominant limb and all were lateral ankle sprain) in the one year prior to the isokinetic evaluation. The isokinetic results demonstrated that the mean peak torque values for eversion of the non-dominant limb were significantly lower in comparison to the dominant limb at $60 \%$. Moreover, although no difference was observed in the average values of the evertor/invertor ratio between the limbs, the ratios were below the values suggested by the literature. Conclusion: We believe that although the athletes reported no ankle injury and no pain in the previous assessment month, the isokinetic results of the non-dominant limb seemed to be directly related to previous ankle sprains injuries in inversion of this limb. The athletes had similar results to those of subjects clinically diagnosed with ankle joint instabilities despite their being active in sports at the moment of the assessment.
\end{abstract}

Keywords: team sport; strength, biomechanics.

\section{Introduction}

Volleyball is one of the most practiced sport worldwide and is characterized by short, intense, and explosive movements; such as jumps, attack hits, blocks, services, passes, and lateral shuffling ${ }^{1}$. However, the most important actions observed during the volleyball practice are the offensive movements (services, blocks, and attack hits) which requires many muscle and joint demands ${ }^{2}$. Since it is a sport with no physical contact and the opponents are separated by a net, volleyball is considered to be a safe sport, and, consequently, there is a low incidence of musculoskeletal injuries when compared to other sports ${ }^{3-4}$. Nevertheless, previous studies have shown that the most frequent injury which affect volleyball players is the ankle sprain ${ }^{2-3,5}$ and that according to a recent meta-analysis by Doherty, Delahunt, Caulfield, Hertel, Ryan, Bleakley ${ }^{6}$ and females have higher chances of ankle sprains.

The main factor related to ankle sprains is the high impact after a vertical jump, which is a fundamental movement and is considered a differential for better performance by the athletes during services, blocks and attack hits ${ }^{7}$. Nevertheless, just the impact intensity alone does not justify the occurrence of these injuries, and the malfunctioning of the structures that comprise this joint, such as the ankle muscles, also justify the high occurrence of ankle sprains ${ }^{8}$.

The study of the ankle muscles is of great importance as it is related to the good performance during volleyball practice and since it is involved in the mechanism of jumping and landing on a single foot, making the ankle one of the most injured joints ${ }^{9}$. The comparison between limbs' strength is important to determine muscular balance because bilateral asymmetries indicates a higher risk of musculoskeletal injuries ${ }^{10}$. Thus, the isokinetic testing, which is an assessment method of high effectivity and trustworthiness, can be used to compare bilateral and agonist/ antagonist muscle strength of the athletes ${ }^{11}$.

Although many studies have used the isokinetic testing in the muscular study of different sports populations, and joints, there are few studies directed to adolescent volleyball athletes, and, especially, to the ankle joint. Therefore, this study aimed to analyze, through information from a database, the muscular performance and differences between the dominant and nondominant limbs of the evertor and invertor muscles of the ankle in adolescent female volleyball players.

\section{Methods}

This quantitative, cross-sectional and retrospective study was conducted at the Instituto de Medicina do Esporte e Ciências Aplicadas ao Movimento Humano da Universidade de Caxias do Sul (IME-UCS) in the city of Caxias do Sul, Rio Grande do Sul, Brazil. It was approved (protocol number 967.527) by the Ethical Research Committee of the Faculdade Cenecista Bento Gonçalves (Bento Gonçalves, Rio Grande do Sul, Brazil), and conducted according to the 2012 Law N $N^{\circ} 466$ of the National Health Council, which approves the guidelines and rules for 
research involving human beings.

For this study, the IME-UCS' database was used to obtain information regarding the concentric isokinetic evaluation of the ankle's evertor and invertor muscles. The study sample included evaluations from 20 adolescents female volleyball players between the ages of 14-17 years old from Universidade de Caxias do Sul's team. The number of included evaluations was conveniently established and, therefore, determined intentionally and not by probability according to the number of available evaluations in the IME-UCS's database. In the first moment, 24 evaluations were selected; however, four evaluations were excluded because IME-UCS' consent term for future publications had not been authorized by the athletes and their legal responsible. A questionnaire was developed by IME-UCS and used to determine age, height, weight, time of volleyball practice, prevalence of injuries and lower limb dominance. The two main points in the questionnaire was to identify the prevalence of injuries and limb dominance. An injury was defined as an accident during the volleyball practice or competition, which prevented the athlete to practice or play for at least one day after the injury. After, the athletes were asked to describe the injury (e.g. muscle, tendonitis, bruises, sprains, fractures or dislocations), anatomic region (e.g. hip, knee, leg, ankle, foot, shoulder, elbow, arm, hand, head or trunk) and if they received physical therapy treatment. Related to the limb dominance, the athletes were asked what lower limb they used for game movements. The mean age of the athletes was 14.90 $( \pm 2.82)$ years, the mean height was $1.68( \pm 0.78)$ meters, the mean weight was $60.70( \pm 13.43)$ kilograms, and the mean body mass index (BMI) was $21.40( \pm 4.21) \mathrm{kg} / \mathrm{m}^{2}$, which is considered normal $^{12}$. The average time of volleyball practice was 3.60 $( \pm 2.21)$ years. Of all the players, 14 had lower musculoskeletal injuries within the period of one year prior to the isokinetic evaluation, whereas 7 had suffered ankle sprains (6 affected the non-dominant limb and all ankle injuries were lateral ankle sprain); 4 had suffered tibialis anterior tendonitis and 3 had muscle injuries ( 2 knee flexors and 1 hip adductor muscles). All athletes received physical therapy treatment and did not have any musculoskeletal complaint at the time of evaluation. With respect to dominance, 19 athletes reported a dominance of the right limb and just 1 reported dominance of the left limb.

The isokinetic evaluations were made with the institution's isokinetic dynamometer (Biodex System $4^{\circledR}$, Biodex Medical Systems, Shieley, New York, USA). The athletes first underwent warmup exercises on a stationary bicycle for 8 minutes with no resistance at moderate velocity (70-80 revolutions per minute). The athletes were then led through the isokinetic dynamometer. The athletes sat on the dynamometer chair with their torsos at positioned $70^{\circ}$, hips and knees flexed at $90^{\circ}$ and the ankle joint was positioned at $10-15^{\circ}$ plantar. Two straps cross-crossing the dorsum of the food held it against the foot plate and the torso, pelvis, and thigh ( $1 / 3$ distal) were stabilized with belts to avoid compensatory movements. The order of the tests was conducted randomly, some times the first evaluated limb was the dominant limb (DL) and some times was the non-dominant limb (NDL). The athletes performed three sub-maximal repetitions $(50 \%$ of their maximum effort) and a previous maximal for each test on the two velocities to familiarize themselves with the procedures and warmup. Protocol during the test demanded 3 maximal repetitions of ankle eversion and inversion on each angular velocity. A 1-minute rest period was set between evaluations of the two velocities, and a 3-minute rest period between DL and NDL evaluations. Athletes were tested by the same examiner with the use of verbal incentives for stimulation and encouragement to their maximum strength.

Isokinetic variables - peak torque (PT, N•m) and the evertor/ invertor ratio $(\%)$ - were used for the analysis. The means values for PT and the evertor/invertor ratio for the ankle joint musculature were evaluated statistically on the SPSS 17.0 software (Statistical Package to Social Science for Windows, New York, USA). To verify the normality of the data distribution, the Shapiro-Wilk test was used, and the mean values for the DL and NDL tests were evaluated with paired version of student's $\mathrm{T}$ test at the significance level of 0.05 . Bilateral asymmetry between DL and NDL was also evaluated using a specific equation. To calculate the bilateral asymmetry of the PT, the difference between the PT of the DL and NDL was divided by the PT of DL and multiplied by 100 . The Evertor/Invertor ratio's bilateral asymmetry was calculated by dividing the difference between the flexor/extensor ratio of the DL and NDL with the flexor/extensor ratio of the DL and the result was multiplied by 100 (flexor/extensor DL - flexor/extensor ratio NDL * 100) ${ }^{13-14}$.

flexor/extensor ratio DL

\section{Results}

The concentric isokinetic data results of the PT of the DL and NDL are presented in Table 1 . At an angular velocity of $60 \%$, the average values for PT ankle evertor muscle showed significant differences between the limbs.

Table 1. Mean and standard deviation values for peak torque of the evertors and invertors of the dominant limb and the non-dominant limb's ankle.

\begin{tabular}{|c|c|c|c|c|}
\hline \multirow[t]{2}{*}{$\begin{array}{l}\text { Angular } \\
\text { Velocities }\end{array}$} & \multicolumn{3}{|c|}{ PT Ankle Evertors $(\mathrm{N} \bullet \mathrm{m})$} & \multirow{2}{*}{$\begin{array}{c}\text { Bilateral } \\
\text { Asymmetry } \\
(\%)\end{array}$} \\
\hline & DL & NDL & “p” & \\
\hline $60 \% / \mathrm{s}$ & $25.08( \pm 5.54)$ & $19.96( \pm 6.62)^{*}$ & 0.022 & 20.41 \\
\hline $180^{\circ} / \mathrm{s}$ & $19.52( \pm 4.95)$ & $17.77( \pm 5.66)$ & 0.067 & 8.96 \\
\hline \multirow[t]{2}{*}{$\begin{array}{l}\text { Angular } \\
\text { Velocities }\end{array}$} & \multicolumn{3}{|c|}{ PT Ankle Invertors $(\mathrm{N} \bullet \mathrm{m})$} & $\begin{array}{c}\text { Bilateral } \\
\text { Asymmetry }\end{array}$ \\
\hline & DL & NDL & “p” & \\
\hline $60 \% / \mathrm{s}$ & $27.88( \pm 8.03)$ & $25.90( \pm 10.77)$ & 0.327 & 7.10 \\
\hline $180^{\circ} / \mathrm{s}$ & $20.62( \pm 5.75)$ & $19.89( \pm 7.17)$ & 0.370 & 3.54 \\
\hline
\end{tabular}

$\mathrm{DL}=$ dominant limb, NDL $=$ non-dominant limb, $\mathrm{PT}=$ peak torque, $*=\mathrm{p}<$ 0.05 . 
Table 2 shows that there was no significant difference between the DL and NDL average value results of the evertor/invertor ratio for the angular velocities of $60 \%$, and $180^{\circ} / \mathrm{s}$.

Table 2. Mean and standard deviation values for the evertor/ invertor ratio of the dominant limb and the non-dominant limb's ankle.

\begin{tabular}{ccccc}
\hline $\begin{array}{c}\text { Angular } \\
\text { Velocities }\end{array}$ & \multicolumn{2}{c}{ Evertor/Invertor ratio (\%) } & $\begin{array}{c}\text { Bilateral } \\
\text { Asymmetry } \\
(\%)\end{array}$ \\
\cline { 2 - 4 } & $\mathbf{D L}$ & NDL & “p” & \\
\hline $60 \% / \mathrm{s}$ & $89.35( \pm 19.37)$ & $90.49( \pm 27.09)$ & 0.929 & -1.27 \\
$180 \% / \mathrm{s}$ & $97.68( \pm 29.73)$ & $90.61( \pm 27.49)$ & 0.329 & 7.24 \\
\hline
\end{tabular}

$\mathrm{DL}=$ dominant limb, $\mathrm{NDL}=$ non-dominant limb.

\section{Discussion}

The purpose of this study was to analyze the peak torque (PT) and the evertor/invertor ratio (Ever/Inver ratio) of the ankle joint in adolescent female volleyball athletes through an isokinetic test. In the analysis of the mean PT values, the NDL presented mean PT values of the evertor muscles significantly lower than the DL at angular velocity of $60 \%$ s. In the analysis at $180 \%$ s, despite the tendency $(\mathrm{p}=0.067)$, there was no statistically significant difference in mean PT values between the limbs as well as in the analysis of the invertor muscles at both velocities. An additional investigation was performed and the results were similar to the statistically analysis. In the bilateral asymmetry analysis, the PT of the evertor muscles at $60 \%$ s was the only result where the difference between the limbs were higher than $15 \%(20.41 \%)$. Although there is no consensus about the values, it has been reported that bilateral differences higher than $10 \%{ }^{15}$ or $15 \%{ }^{16}$ indicates imbalances between the limbs and may increase the risk of injuries and reinjuries in sports $^{15-16}$. Even though studies about bilateral asymmetry of ankle's muscle are rare ${ }^{17}$, it is an important parameter for the rehabilitation process and injury prevention ${ }^{18}$.

Two classic studies on the isokinetic assessment of healthy subjects of both genders ${ }^{19-20}$ with no previous ankle sprain performed at angular velocities of $30 \% \mathrm{~s}, 60 \% \mathrm{~s}$, and $120 \% \mathrm{~s}$, demonstrated no PT differences for evertor and invertors, except for the evertors at $30 \%$ in the study by Wong, Glasheen-Way, Andrews ${ }^{19}$. More recent, study by Lin, Liu, Hsieh, Lee ${ }^{17}$ also found no statistically significant differences in healthy subjects. Interestingly, the 7 episodes of ankle sprains reported by the athletes were sprains in inversion and 6 of them affected the NDL - factors which might be directly related to the isokinetic testing results. The assessed athletes indicated that ankle sprain was the most common injury, representing $50 \%$ of the reported injuries in the previous year. This result corroborates with findings from other studies that have also shown a high rate of ankle injuries representing approximately half of the musculoskeletal injuries in female volleyball athletes ${ }^{2,5,21}$. The athletes reported that these sprains occurred laterally; an injury mechanism which can be defined as a traumatic injury resulted from an excessive inversion of the ankle ${ }^{22}$. During the inversion of the ankle, a reflexive contraction of the evertor muscles occurs ${ }^{23}$ proving the important role of this muscle group in the dynamic stabilization of this joint against the inverting forces ${ }^{24}$. However, the systematic review by de Oliveira Menacho, Pereira, de Oliveira, Chagas, Toyohara, Cardoso $^{25}$ demonstrated that after an episode of ankle sprain there is a decrease of the neuromuscular reflex; resulting in a significantly lower PT of the evertor muscles when compared to not injured joints. Perron, Moffet, Nadeau, Hébert, Belzile ${ }^{26}$ showed that after only one episode of inversion ankle sprain, the evertor weakness persists up to 6 months after the injury.

Regarding the Ever/Inver ratio, which represents the proportion of strength of the ankle evertors in relation to the invertors, the results showed no statistically significant differences and no bilateral asymmetry between DL and NDL at both angular velocities. The assessment of healthy subjects with no previous ankle injury ${ }^{17,27}$ also showed no difference between limbs in relation to the Ever/Inver ratio. However, mean values of the Ever/Inver ratio in this study range from $89.35 \%$ to $97.68 \%$, values below $100 \%$ suggested by literature ${ }^{28}$. Other studies on healthy subjects demonstrated mean values of the Ever/Inver ratio ranging from $103 \%$ to $120 \%{ }^{17,27,29}$. Nevertheless, values below $100 \%$ were also found in the assessment of healthy women ${ }^{20}$. Mean values above $100 \%$, besides demonstrating that the evertor muscles have PT values higher than the invertor muscles, also indicate that the invertor muscles have a greater functional capacity of stabilizing the ankle joint ${ }^{30}$. According to Ersoz, Atalay, Kumbara, $\mathrm{Akyuz}^{27}$, although the investigation of the PT mean values is important, the analysis of the Ever/ Inver ratio is the most essential parameter to be assessed and it might easily demonstrate the imbalance and the impaired muscle group.

The asymmetry of the PT mean values between limbs and the Ever/Inver ratio below normality suggested by the literature might be explained due to various factors, mainly due to an previous ankle sprain ${ }^{5}$. Ankle sprains in inversion predisposes subjects to other consequent sprains which can be twice more frequent when compared to a subject with no previous injury ${ }^{31}$. According to a literature review by Calatayud, Borreani, Colado, Flandez, Page, Andersen ${ }^{32}$, the increased risk of new sprains often results from remaining structural and functional alterations from prior injury. Among the functional factors, small strength deficits of the evertor muscles weakness can alter the dynamic joint stabilization ${ }^{33-34}$. If structural and functional recovery do not occur, new sprain episodes will lead to functional instability or chronic instability of the ankle joint, and, consequently, will further accentuate the evertor muscles weakness ${ }^{33-35}$. In addition to the evertor muscles deficits, alterations in proprioception, in neuromuscular control, and in postural control may be involved in functional deficits of the ankle ${ }^{36-37}$. In order to recovery the muscle strength and 
proprioception, professionals of rehabilitation has to focus on evertor muscle after an ankle sprain ${ }^{38}$.

\section{Conclusion}

This study demonstrated that the evertor muscles of NDL had lower PT values than DL and mean values of Ever/Inver ratio were below normality for both limbs. Although the athletes reported no ankle injury and no pain in the previous assessment month, the isokinetic results of the non-dominant limb seemed to be directly related to previous lateral ankle injuries that affected 6 athletes' non-dominant limbs. The results has a great clinical importance since it was possible to show the athletes and the team staff about the importance of enhancing muscular performance during and after the rehabilitation process. It is important in order to reach the bilateral balance between the limbs, both for athletic performance and for minimizing chances of subsequent ankle sprains. However, new studies with larger samples performed at different angular velocities and different types of muscular contractions are necessary to contribute with scientific knowledge concerning the evertor and invertor ankle muscles performance.

\section{References}

1. Mroczek D, Januszkiewicz A, KawczyNski AS, Borysiuk Z, Chmura J. Analysis of male volleyball players' motor activities during a top level match. J Strength Cond Res. 2014;28(8):2297-305.

2. Bere T, Kruczynski J, Veintimilla N, Hamu Y, Bahr R. Injury risk is low among world-class volleyball players: 4-year data from the FIVB Injury Surveillance System. Br J Sports Med. 2015;49(17):1132-7.

3. Beneka A, Malliou P, Gioftsidou A, Tsigganos G, Zetou H, Godolias G. Injury incidence rate, severity and diagnosis in male volleyball players. Sport Sci Health. 2009;5(3):93-9.

4. Reeser JC, Joy EA, Porucznik CA, Berg RL, Colliver EB, Willick SE. Risk factors for volleyball-related shoulder pain and dysfunction. PM R. 2010;2(1):27-36.

5. Verhagen E, Van der Beek A, Bouter L, Bahr RM, Van Mechelen W. A one season prospective cohort study of volleyball injuries. Br J Sports Med. 2004;38(4):477-81.

6. Doherty C, Delahunt E, Caulfield B, Hertel J, Ryan $\mathrm{J}$, Bleakley $\mathrm{C}$. The incidence and prevalence of ankle sprain injury: a systematic review and meta-analysis of prospective epidemiological studies. Sports Med. 2014;44(1):123-40.

7. Borràs X, Balius X, Drobnic F, Galilea P. Vertical jump assessment on volleyball: a follow-up of three seasons of a high-level volleyball team. J Strength Cond Res. 2011;25(6):1686-94.

8. Delahunt E, Monaghan K, Caulfield B. Altered neuromuscular control and ankle joint kinematics during walking in subjects with functional instability of the ankle joint. Am J Sports Med. 2006;34(12):1970-6.

9. Suda EY, Amorim CF, Sacco IDCN. Influence of ankle functional instability on the ankle electromyography during landing after volleyball blocking. J Electromyogr Kinesiol. 2009;19(2):e84-93.

10. Schlumberger A, Laube W, Bruhn S, Herbeck B, Dahlinger M, Fenkart G, et al. Muscle imbalances - fact or fiction? Isokinetics Exer Sci. 2006;14(1):3-11.

11. Jones PA, Bampouras TM. A comparison of isokinetic and functional methods of assessing bilateral strength imbalance. J Strength Cond Res. 2010;24(6):1553-8.

12. World Healthy Organization (WHO). Global Health Observatory data repository. Available at: http://apps.who. int/gho/data/node.main.A897A?lang=en [Acessed 19th February 2018].

13. Chavet P, Lafortune MA, Gray JR. Asymmetry of lower extremity responses to external impact loading. Hum Mov Scie. 1997;16(4):391-06.

14. Silva JRLC, Detanico D, Dal Pupo J, Freitas CDL. Bilateral asymmetry of knee and ankle isokinetic torque in soccer players U20 category. Rev Bras Cineantropom Desempenho Hum. 2015;17(2):195-204.

15. Brumitt J, Heiderscheit BC, Manske RC, Niemuth PE, Rauh MJ. Lower extremity functional tests and risk of injury in division III collegiate athletes. Int J Sports Phys Ther. 2013;8(3):216-27.

16. Fousekis K, Tsepis E, Vagenas G. Lower limb strength in professional soccer players: profile, asymmetry, and training age. J Sports Sci Med. 2010;9(3):364-73

17. Lin WH, Liu YF, Hsieh CCC, Lee AJ. Ankle eversion to inversion strength ratio and static balance control in the dominant and non-dominant limbs of young adults. J Sci Med Sport. 2009;12(1):42-9.

18. Willems T, Witvrouw E, Verstuyft J, Vaes P, De Clercq D. Proprioception and muscle strength in subjects with a history of ankle sprains and chronic instability. J Athl Train. 2002 37(4);487-93.

19. Wong DL, Glasheen-Way M, Andrews LF. 1sokinetic evaluation of the ankle invertors and evertors. J Orthop Sports Phys Ther. 1984;5(5):246-52.

20. Nickson W. Normative isokinetic data on the ankle invertors and evertors. Aust J Physiother. 1987;33(2):85-90.

21. Malliou P, Beneka A, Tsigganos G, Gioftsidou A, Germanou E, Michalopoulou M. Are injury rates in female volleyball players age related? Sport Sci Health. 2008;2(3):113-7.

22. Delahunt E, Coughlan GF, Caulfield B, Nightingale EJ, Lin CWC, Hiller CE. Inclusion criteria when investigating insufficiencies in chronic ankle instability. Med Sci Sports Exerc. 2010;42(11):2106-21.

23. Knight AC, Weimar WH. Difference in ratio of evertor to invertor activity between the dominant and nondominant legs during simulated lateral ankle sprain. J Sport Rehabil. 2013;22(4):272-8. 
24. Wright CJ, Arnold BL. Fatigue's effect on eversion force sense in individuals with and without functional ankle instability. J Sport Rehabil. 2012;21(2):127-36.

25. de Oliveira Menacho M, Pereira HM, de Oliveira BIR, Chagas LMPM, Toyohara MT, Cardoso JR. The peroneus reaction time during sudden inversion test: systematic review. J Electromyogr Kinesiol. 2010;20(4):559-65.

26. Perron M, Moffet H, Nadeau S, Hébert LJ, Belzile S. Persistence of long term isokinetic strength deficits in subjects with lateral ankle sprain as measured with a protocol including maximal preloading. Clin Biomech. 2014;29(10):1151-7.

27. Ersoz M, Atalay NS, Kumbara F, Akyuz M. Investigation of effect of age, gender and limb dominance on ankle evertor/invertor peak torque ratios of healthy volunteers. J Phys Ther Sci. 2009;21(3):263-7.

28. Hartsell HP, Spaulding SJ. Eccentric/concentric ratios at selected velocities of the invertors and evertors muscles of the chronically unstable ankle. Br J Sports Med. 1999;33(4):255-8.

29. Yüksel O, Ozgürbüz C, Ergün M, İşlegen, Ç, Taskiran E, Denerel N, et al. Inversion/Eversion strength dysbalance in patients with medial tibial stress syndrome. J Sports Sci Med. 2011;10(4):737-42.

30. Kaminski T, Buckley B, Powers M, Hubbard TJ, Ortiz C. Effect of strength and proprioception training on eversion to inversion strength ratios in subjects with unilateral functional ankle instability. Br J Sports Med. 2003;37(5):410-5.

31. McHugh MP, Tyler TF, Tetro DT, Mullaney MJ, Nicholas SJ. Risk factors for noncontact ankle sprains in high school athletes: the role of hip strength and balance ability. Am J Sports Med. 2006;34(3):464-70.

32. Calatayud J, Borreani S, Colado JC, Flandez J, Page P, Andersen LL. Exercise and ankle sprain injuries: a comprehensive review. Phys Sportsmed. 2014;42(1):88-93.

33. Terrier R, Rose-Dulcina K, Toschi B. Impaired control of weight bearing ankle inversion in subjects with chronic ankle instability. Clin Biomech. 2014;29(4):439-43.
34. Cameron KL, Owens BD, DeBerardino TM. Incidence of ankle sprains among active-duty members of the United States armed services from 1998 through 2006. J Athl Train. 2010;45(1):29-38.

35. Arnold BL, Linens SW, De la Motte SJ, Ross SE. Concentric evertor strength differences and functional ankle instability: a meta-analysis. J Athl Train. 2009;44(6):653-62.

36. Zech A, Hübscher M, Vogt L, Banzer W, Hänsel F, Pfeifer, K. Neuromuscular training for rehabilitation of sports injuries: a systematic review. Med Sci Sports Exer. 2009;41(10):1831-41.

37. Hanci E, Sekir U, Gur H, Akova B. Eccentric training improves ankle evertor and dorsiflexor strength and proprioception in functionally unstable ankles. Am J Phys Med Rehabil. 2016;95(6):448-58.

38. Collado H, Coudreuse JM, Graziani F, Bensoussan L, Viton JM, Delarque A. Eccentric reinforcement of the ankle evertor muscles after lateral ankle sprain. Scand J Med Sci Sports. 2010;20(2):241-6.

\section{Corresponding author}

Leandro Viçosa Bonetti, PT, PhD, Professor of Physical Therapy,

Universidade de Caxias do Sul, RS, Brazil.

Rua Francisco Getúlio Vargas, 1130, Bloco 70, Caxias do Sul, RS, Brasil, CEP 95070-560.

Email: leandrovbonetti@gmail.com / lvbonetti@ucs.br

Manuscript received on March 26, 2018

Manuscript accepted on April 11, 2018

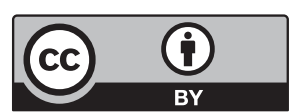

Motriz. The Journal of Physical Education. UNESP. Rio Claro, SP, Brazil

- eISSN: 1980-6574 - under a license Creative Commons - Version 3.0 